\title{
A Mode-Matching Solution for TE-Backscattering from an Arbitrary 2D Rectangular Groove in a PEC
}

\author{
Mehdi Bozorgi*
}

\begin{abstract}
In this paper, the simple yet effective mode-matching technique is utilized to compute TE-backscattering from a $2 \mathrm{D}$ filled rectangular groove in an infinite perfect electric conductor (PEC). The tangential magnetic fields inside and outside of the groove are represented as the sums of infinite series of cosine harmonics (half-range Fourier cosine series). By applying the continuity of the tangential magnetic field, these modes are matched on the groove to obtain the series coefficients by solving a system of linear equations. For this purpose, some oscillatory logarithmic singular integrals involving Hankel and trigonometric functions are solved numerically, starting by removing the logarithmic singularity via integration by parts. In the following, the new well-behaved highly oscillatory integrals are computed using efficient methods, and several comparisons are made to demonstrate the validity and ability of the presented procedure.
\end{abstract}

Key Words: Highly Oscillatory Singular Integral, Modal Expansion, TE-Backscattering, 2D Filled Rectangular Groove.

\section{INTRODUCTION}

Electromagnetic wave scattering from a groove has received attention due to its broad applicability and easy realization in radar cross-section and non-destructive testing applications [1, 2]. An analytical solution for a narrow groove is given in the literature [3], and the finite element and hybrid numerical techniques have been investigated to simulate scattering from an arbitrary shape groove $[4,5]$. To study scattering from a large crack, a Fourier transform was proposed in [6], and, for narrow and wide rectangular cracks, a complex direct integral equation solver (DIES) was proposed to solve a singular integral equation by a collocation method based on Chebyshev polynomials [2]. From the base modal technique, other techniques were developed to efficiently calculate electromagnetic waves backscattered from grooves [7-10], which considered some simplifications and assumptions to avoid solving complicated singular integrals.
Morgan and Schwering [7] used the physical optic approximation to model equivalent electric current distributions on the groove and considered two synthetic waveguide walls in upper space; this creates significant error for narrow grooves but is somewhat accurate for wide apertures. As reported in [8], the constructed integral equation in the spectral domain is converted to a system of linear algebraic equations to compute the unknown Rayleigh function. To improve the computational efficiency of this technique, a model-based parameter estimation technique based on a curve-fitting model was employed. In [9], by applying Fourier transform techniques to a $2 \mathrm{D}$ groove, the singularity of the constructed integral could be analytically removed and an exact expression for the far-field scattering obtained by stationary phase approximation. Cho [10] also employed the overlapping T-block method to obtain an accurate closed-form expression for far-field scattered waves.

In this paper, an approach based on the usual mode-mat-

Manuscript received May 11, 2019 ; Revised June 16, 2019 ; Accepted September 11, 2019. (ID No. 20190511-033J)

Department of Electrical Engineering, Faculty of Engineering, Arak University, Arak, Iran.

"Corresponding Author: Mehdi Bozorgi (e-mail: M-Bozorgi@araku.ac.ir)

This is an Open-Access article distributed under the terms of the Creative Commons Attribution Non-Commercial License (http://creativecommons.org/licenses/by-nc/4.0) which permits unrestricted non-commercial use, distribution, and reproduction in any medium, provided the original work is properly cited.

(c) Copyright The Korean Institute of Electromagnetic Engineering and Science. All Rights Reserved. 
ching methodology is developed, without the limiting assumptions made in [7] and away from the complex expressions presented in [2]. It is used to obtain the equivalent magnetic current on the groove, which can create the scattered fields. At first, the fields are represented as a sum of infinite harmonic modes in the open rectangular cavity. Applying the equivalence principle, an unknown equivalent magnetic current is simulated over the groove, which is then calculated. Mode-by-mode field matching across the groove then leads to an infinite system of linear equations. While allowing sufficient accuracy, the infinite sums are truncated to obtain the expansion coefficients. To expand the tangential magnetic field in the upper half-space outside the groove, a highly oscillatory singular integral should be solved. There are some quadrature techniques to calculate singular logarithmic integrals $[11,12]$, most of which assume that the singular integral contains a logarithmic singular part and functions with slow variations. However, if a singular integral includes trigonometric and Bessel functions simultaneously with large arguments, the number of quadrature nodes must increase significantly and, therefore, the simulation time increases immensely. For this reason, advanced techniques have been developed to compute a highly oscillatory integral quickly. Here, in the first stage, the singularity is eliminated through integration by parts; the remaining oscillatory integrals can then be calculated quickly with sufficient precision using the efficient methods in [13-15]. This solution is valid for narrow and wide grooves, with the advantages of accuracy and acceptable time consumption, particularly for the wide grooves.

\section{FORMULATION}

Assume a TE electromagnetic plane wave,

$$
H_{z}^{i}=\vec{z} e^{j k_{1}\left(\left(x-\frac{w}{2}\right) \cos \varphi_{0}+y \sin \varphi_{0}\right)} \quad \varphi_{0} \in(0, \pi) .
$$

where $k_{1}$ is the free space propagation constant and $\varphi_{0}$ is the incidence angle. As shown in Fig. 1, the wave illuminates a dielectric-filled 2D rectangular groove with $\varepsilon_{2}$ and $\mu_{2}$.

The tangential magnetic field inside the groove is expanded by cosine basis functions to satisfy Maxwell's equations [7].

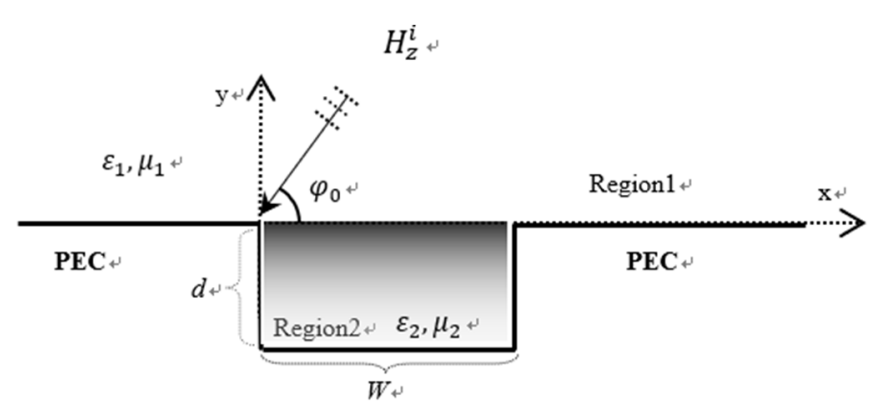

Fig. 1. The geometry of the rectangular groove and TE-mode incident wave.
Thus, the $\mathrm{x}$-component of the electric field inside the groove can be obtained as follows:

$$
\begin{aligned}
& E_{x}(x, y)=\frac{\partial H_{z}(x, y)}{\partial y} \\
& \quad=\sum_{p=0}^{\infty} A_{p} k_{p} \cos \left(\frac{p \pi x}{W}\right) \times \sin \left(k_{p}(y+d)\right)
\end{aligned}
$$

where $k_{p}=\sqrt{k_{2}^{2}-\left(\frac{p \pi}{w}\right)^{2}}$ and $A_{p}$ are unknown series coefficients that should be calculated. By invoking the equivalence principle on the groove, the magnetic current $\vec{M}$ at $y=0$ is calculated to be

$$
\begin{aligned}
\vec{M}(x) & =\left[E_{x}(x) \vec{x}+E_{y}(x) \vec{y}\right] \times \vec{y}=E_{x}(x) \vec{z} \\
& =\vec{z} \sum_{p=0}^{\infty} A_{p} k_{p} \cos \left(\frac{p \pi x}{W}\right) \sin \left(k_{p} d\right)
\end{aligned}
$$

Considering Green's functions in two regions (Region1, free space; Region2, groove), the tangential magnetic fields resulting from the magnetic current $M_{z}(x)$ are obtained as follows [2]:

$$
\begin{aligned}
& H_{z}^{\text {Region } 1}(x)=-\frac{k_{1} y_{1}}{2} \sum_{p=0}^{\infty} A_{p} k_{p} \sin \left(k_{p} d\right) \\
& \times \int_{0}^{W} \cos \left(\frac{p \pi x^{\prime}}{W}\right) H_{0}^{(2)}\left(k_{1}\left|x-x^{\prime}\right|\right) d x^{\prime}
\end{aligned}
$$

and

$$
\begin{gathered}
H_{z}^{\text {Region } 2}(x)=\frac{j k_{2} y_{2}}{W} \sum_{p=0}^{\infty} A_{p} k_{p} \sin \left(k_{p} d\right) \\
\times \sum_{n=0}^{\infty} \frac{\varepsilon_{n}}{k_{n} \tan \left(k_{n} d\right)} \cos \left(\frac{n \pi x}{W}\right) \int_{0}^{W} \cos \left(\frac{n \pi x^{\prime}}{W}\right) \cos \left(\frac{p \pi x^{\prime}}{W}\right) d x^{\prime}
\end{gathered}
$$

where $y_{1}$ and $y_{2}$ are the intrinsic admittances for Region1 and Region2, respectively; $k_{n}=\sqrt{k_{2}^{2}-\left(\frac{n \pi}{w}\right)^{2}} ; H_{0}^{(2)}($.$) is the$ zeroth-order Hankel function of the second kind; and $\varepsilon_{n}=$ 1 if $n=0$ and $\varepsilon_{n}=2$ otherwise. By imposing the continuity of the tangential magnetic field on both sides of the groove $(y=0)$, we have

$$
2 e^{j k_{1}\left(x-\frac{W}{2}\right) \cos \varphi_{0}}+H_{z}^{\text {Region } 1}(x)=H_{z}^{\text {Region } 2}(x) .
$$

Substituting (4)-(5) into (6) and simplifying, we construct the following equation:

$$
\begin{gathered}
2 e^{j k_{1}\left(x-\frac{W}{2}\right) \cos \varphi_{0}}-\frac{k_{1} y_{1}}{2} \sum_{p=0}^{\infty} A_{p} k_{p} \sin \left(k_{p} d\right) \quad Q_{p}(x) \\
=-j k_{2} y_{2} \sum_{p=0}^{\infty} A_{p} \cos \left(k_{p} d\right) \cos \left(\frac{p \pi x}{W}\right)
\end{gathered}
$$

where the function $Q_{p}(x)$ is defined as

$$
Q_{p}(x)=\int_{0}^{W} \cos \left(\frac{p \pi x^{\prime}}{W}\right) H_{0}^{(2)}\left(k_{1}\left|x-x^{\prime}\right|\right) d x^{\prime} .
$$

In Eq. (7), to utilize the mode-matching technique, the function $Q_{p}(x)$ and the function $\exp \left(j k_{1}\left(x-\frac{W}{2}\right)\right) \cos \varphi_{0}$ should 
be expanded using half-range Fourier cosine series, i.e.,

$$
Q_{p}(x)=\sum_{k=0}^{\infty} q_{p k} \cos \left(\frac{k \pi x}{W}\right)
$$

and

$$
2 e^{j k_{1}\left(x-\frac{W}{2}\right) \cos \varphi_{0}}=\sum_{p=0}^{\infty} b_{p} \cos \left(\frac{p \pi x}{W}\right) .
$$

The coefficients $b_{p}$ in (10) can be easily calculated analytically as

$$
b_{p}=\varepsilon_{p} \alpha \frac{(-1)^{p} e^{\alpha / 2}-e^{-\alpha / 2}}{(p \pi)^{2}+\alpha^{2}}
$$

where $\alpha=j k_{1} W \cos \varphi_{0}$ and $\varepsilon_{p}$ is like $\varepsilon_{n}$ in (5). The coefficients $q_{p k}$ in (9) can also be computed as

$$
\begin{aligned}
q_{p k}=\frac{\varepsilon_{p}}{W} \int_{0}^{W} \int_{0}^{W} \cos \left(\frac{k \pi x}{W}\right) \cos \left(\frac{p \pi x^{\prime}}{W}\right) \\
\times H_{0}^{(2)}\left(k_{1}\left|x-x^{\prime}\right|\right) d x^{\prime} d x
\end{aligned}
$$

The integral in (12) - the so-called highly oscillatory singular integral—cannot be computed easily. It should, however, be noted that, when the values of $k, p$, and $W$ increase, we need a large number of quadrature nodes to maintain accuracy, and the computing time increases enormously. Some rapid methods have been reported in [13-15] to calculate these integrals numerically. To solve the integral specified in (12), let us first focus on the following highly oscillatory integral:

$$
q_{p k}=\frac{\varepsilon_{p}}{W} \int_{0}^{W} \cos \left(\frac{k \pi x}{W}\right) Q_{p}(x) d x
$$

Introducing the variable $\xi=\frac{2}{W} x-1$ in interval $[-1,1]$, the integral (13) is split up into two cosine and sine integrals:

$$
q_{p k}=\left\{\begin{array}{l}
\frac{\varepsilon_{p}}{2}(-1)^{\frac{k}{2}} \int_{-1}^{1} \cos \left(\frac{k \pi}{2} \xi\right) Q_{p}(\xi) d \xi k: \text { even } \\
\frac{\varepsilon_{p}}{2}(-1)^{\frac{k+1}{2}} \int_{-1}^{1} \sin \left(\frac{k \pi}{2} \xi\right) Q_{p}(\xi) d \xi k: \text { odd }
\end{array}\right.
$$

Here, we assume $Q_{p}(\xi)$ is a function with a removable singularity part. A quadrature-based Legendre polynomials technique is used to evaluate the highly oscillatory integrals in (14) [13]. Therefore, the above integrals can be calculated via the following integral:

$$
\begin{array}{r}
\int_{-1}^{1} e^{\frac{j k \pi}{2} \xi} Q_{p}(\xi) d \xi=\sum_{m=0}^{M} j^{m}\left[\frac{(2 m+1)^{2}}{m}\right]^{\frac{1}{2}} \\
\times J_{m+\frac{1}{2}}\left(\frac{k \pi}{2}\right) \sum_{s=0}^{M} w_{s} P_{m}\left(\xi_{s}\right) Q_{p}\left(\xi_{s}\right)
\end{array}
$$

where $w_{s}$ and $\xi_{s}$ are weights and abscissas (defined as the roots of the Legendre polynomial), respectively, of the $(M+1)$ point Gauss-Legendre rule [13], and $J_{m+\frac{1}{2}}($.$) and P_{m}($.$) are-$ a Bessel function and Legendre polynomial of degree $m$, respecttively. Now, by presenting $\xi^{\prime}=\frac{2}{W} x^{\prime}-1$, integral $Q_{p}\left(\xi_{s}\right)$ in (15) can be arranged in the form

$$
\begin{aligned}
Q_{p}\left(\xi_{s}\right) & =\frac{W}{2} \int_{-1}^{1} \cos \left(\frac{p \pi}{2}\left(1+\xi^{\prime}\right)\right) \\
& \times H_{0}^{(2)}\left(\frac{k_{1} W}{2}\left|\xi_{s}-\xi^{\prime}\right|\right) d \xi^{\prime}
\end{aligned}
$$

The integral in (16) contains the highly oscillatory and logarithmic functions (Henkel and cosine function). In computing $Q_{p}\left(\xi_{s}\right)$, regular approaches generally do not lead to a proper result. At this point, to eliminate the logarithmic singularity of the zeroth-order Hankel function of the second kind at $\xi^{\prime}=\xi_{s}$, direct integration by parts is suggested. The integral in (16) is written in the following form:

$$
\begin{gathered}
Q_{p}\left(\xi_{s}\right)=-\frac{W}{2} \times \\
{\left[\left(\xi_{s}-\xi^{\prime}\right) \cos \left(\frac{p \pi}{2}\left(1+\xi^{\prime}\right)\right) H_{0}^{(2)}\left(\frac{k_{1} W}{2}\left|\xi_{s}-\xi^{\prime}\right|\right)\right]_{-1}^{1}} \\
-\frac{p \pi W}{4} \int_{-1}^{1} \sin \left(\frac{p \pi}{2}\left(1+\xi^{\prime}\right)\right)\left(\xi_{s}-\xi^{\prime}\right) \\
\times H_{0}^{(2)}\left(\frac{k_{1} W}{2}\left|\xi_{s}-\xi^{\prime}\right|\right) d \xi^{\prime}+\frac{k_{1} W^{2}}{4} \times \\
\int_{-1}^{1} \cos \left(\frac{p \pi}{2}\left(1+\xi^{\prime}\right)\right) \frac{\left(\xi_{s}-\xi^{\prime}\right)^{2}}{\left|\xi_{s}-\xi^{\prime}\right|} H_{1}^{(2)}\left(\frac{k_{1} W}{2}\left|\xi_{s}-\xi^{\prime}\right|\right) d \xi^{\prime}
\end{gathered}
$$

As seen in (17), at $\xi^{\prime}=\xi_{s}$, the resulting integrals are not singular, but rather still consist of highly oscillatory functions. They can be computed in the same manner proposed for the integrals in (14). We can also use the rapid numerical methods developed for integrals containing Bessel and trigonometric functions $[14,15]$. After calculating $q_{p k}$ and substituting (9)(10) into (7), Eq. (7) can finally be rearranged as follows:

$$
\begin{aligned}
& \sum_{p=0}^{\infty} b_{p} \cos \left(\frac{p \pi x}{W}\right) \\
& \quad-\sum_{k=0}^{\infty} \sum_{p=0}^{\infty} A_{p} \frac{k_{1} y_{1}}{2} q_{p k} k_{p} \sin \left(k_{p} d\right) \cos \left(\frac{k \pi x}{W}\right) \\
& =-\sum_{p=0}^{\infty} A_{p} j k_{2} y_{2} \cos \left(k_{p} d\right) \cos \left(\frac{p \pi x}{W}\right)
\end{aligned}
$$

While allowing sufficient accuracy, infinite sums in (18) are truncated at $k=K$ and $p=P$. Taking $K=P$, we obtain the following linear system of equations:

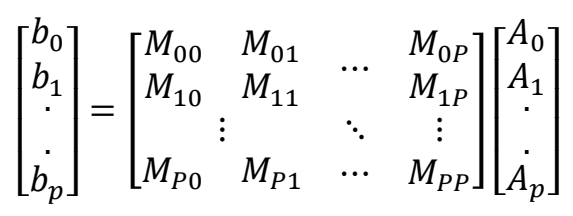

where the elements $M_{p k}$ are given by

$$
\begin{gathered}
M_{p k}= \\
\left\{\begin{array}{c}
\frac{k_{1} y_{1}}{2} q_{p k} k_{p} \sin \left(k_{p} d\right)-j k_{2} y_{2} \cos \left(k_{p} d\right), k=p \\
\frac{k_{1} y_{1}}{2} q_{p k} k_{p} \sin \left(k_{p} d\right), k \neq p
\end{array}\right.
\end{gathered}
$$


The unknown expansion coefficients $A_{p}$ are now obtained by a simple matrix inversion operation. As we know the magnetic current distribution on the aperture, the backscattered fields and the echo width are estimated as in [2]:

$$
\begin{aligned}
H_{z}^{S}= & -\vec{z} \frac{e^{-j k_{0} \rho}}{\sqrt{\rho}} e^{j \frac{\pi}{4}} \frac{k_{1} y_{1}}{2} \\
& \times \sqrt{\frac{2}{\pi k_{1}}} \int_{0}^{W} M_{z}\left(x^{\prime}\right) e^{j k_{1} x^{\prime} \cos \varphi_{0}} d x^{\prime}
\end{aligned}
$$

and

$$
\sigma_{T E}^{2 D}=\lim _{\rho \rightarrow \infty} 2 \pi \rho \frac{\left|H_{Z}^{S}\right|^{2}}{\left|H_{Z}^{i}\right|^{2}}
$$

\section{RESULTS}

Using the above derivations, some comparisons are made to show the effectiveness and accuracy of the suggested procedure. Fig. 2 shows the equivalent magnetic current distribution over a groove with specifications define. As shown in Fig. 2, the obtained results are in good agreement with both DIES [2] and FEKO simulator-based methods of moments. In this case, for the same accuracy, the infinite series in Eq. (18) is truncated at $K=P=15$, while we should use $N=30$ (Chebyshev polynomials degree $N$ ) in the DIES method [2]. To simulate a 2D groove using FEKO software, we use an infinite 3D rectangular trough. To obtain the equivalent magnetic current, the $\mathrm{x}$-component of the near electric field on the middle of the groove $(y=0)$ should be computed. We also utilize a one-dimension periodic boundary condition to simulate the infinite length of the groove. We set frequency $f=29,979 \times 10^{4}$ and $\lambda=1$ to define the required variables and the mesh sizes in wavelength. To provide a highly efficient solution, the object surfaces partially mesh through two local mesh sizes. The local mesh size on the ground plane is set at $0.3 \lambda$, while the minimum values of the triangle edge lengths for the groove surfaces are set to $0.05 d \lambda$ and $0.05 W \lambda$. Fig. 3 shows a comparison between several numerical techniques for predicting backscatter echo width in a wide range of groove widths. Here, a slight amount of loss was added to the filling dielectric $\varepsilon_{2}=\varepsilon_{0}(2-0.01 j), \mu_{2}=\mu_{0}(1-0.01 j)$ to remove

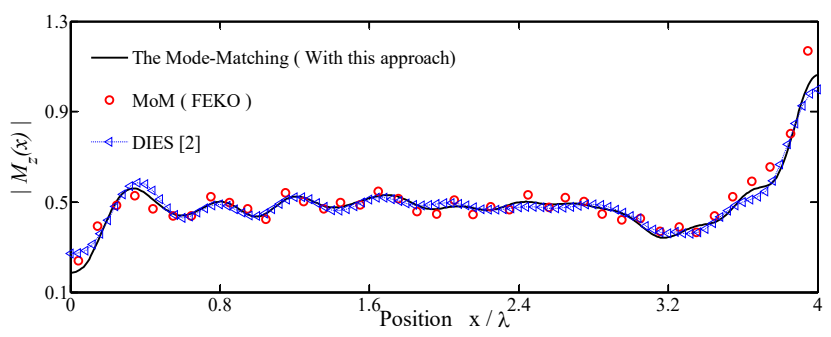

Fig. 2. The magnetic current density for a filled groove $\left(\varepsilon_{r 2}=\right.$ $2.5-j 0.2, \mu_{r 2}=1.8-j 0.1$, and $\left.\varepsilon_{1}=\varepsilon_{0}, \mu_{1}=\mu_{0}\right)$ of $W=4 \lambda$ and $d=0.5 \lambda$ at $\varphi_{0}=45^{\circ}$.

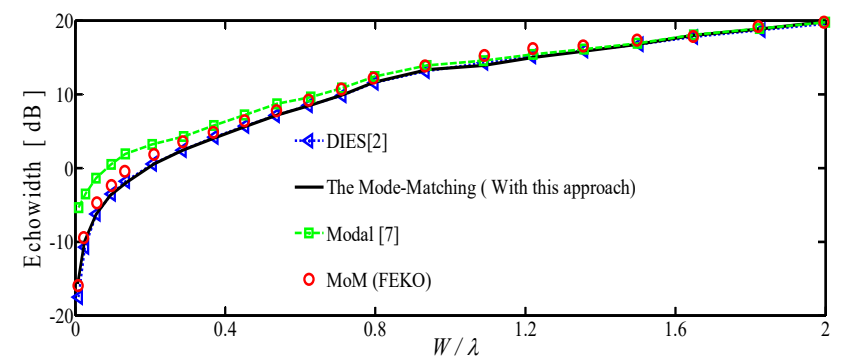

Fig. 3. TE-backscattering echo width versus the groove width for $d=0.2 \lambda, \varphi_{0}=90^{\circ}, \varepsilon_{1}=\varepsilon_{0}, \varepsilon_{2}=2 \varepsilon_{0}-0.01 i$, and $\mu_{1}=\mu_{2}=\mu_{0}$.

the resonances appearing in the echo width graph reported in [10], which happens when $\cos \left(k_{p} d\right)$ in (18) approaches zero.

From Fig. 3, it can be deduced that the suggested procedure is accurate for both wide and narrow grooves, whereas the modal method in [7] is only appropriate for wide grooves. Using the physical optics approximation and replacing the upper halfspace with a waveguide in [7] creates many errors for narrow grooves. To compare the time consumed, we measured the simulation time of each method (for a filled groove with the specification shown below, Fig. 3). The results are presented in Table 1.

By examining the results in Table 1, we can say that, for wide grooves, the modal method presented in [7] is faster than all numerical methods. When the groove width increases, the equivalent magnetic current on the aperture becomes oscillatory. In the DIES method, we need higher-degree Chebyshev polynomials and more quadrature nodes to estimate the rough magnetic current, but it can be simulated by a lower number of harmonic cosine modes. This is why the simulation time of the proposed solution is lower than the DIES method for $W=4 \lambda$. In the previous example, we also employed the technique mentioned in [11] to calculate the double integral in Eq. (12). In this case, the simulation times for $W=0.1 \lambda, W=\lambda$, and $W=4 \lambda$ were measured as $5.21,9.87$, and 73.22 seconds, respectively. A comparison of the above results with the results in Table 1 demonstrates that using advanced numerical integration methods for wide grooves improves time efficiency.

Briefly, the proposed procedure uses the mode-matching

Table 1. Simulation time (in second) of the several methods measured for three groove widths

\begin{tabular}{ccccc}
\hline \multicolumn{1}{c}{ Size } & FEKO $^{\mathrm{a}}$ & Modal [7] & DIES [2] & $\begin{array}{c}\text { Mode-matching } \\
\text { (this approach) }\end{array}$ \\
\hline$W=0.1 \lambda$ & 8 & Not accurate & 1.56 & 5.23 \\
$W=\lambda$ & 25 & 5.07 & 4.58 & 9.14 \\
$W=4 \lambda$ & 52 & 7.07 & 31.20 & 20.36 \\
\hline
\end{tabular}

${ }^{\mathrm{a}}$ Measured in minute. 
method as seen in [7] without any restrictive assumptions. It is accurate for every groove size with acceptable time efficiency.

\section{CONCLUSION}

In this work, a simple solution is suggested to calculate TEbackscattering from an arbitrary rectangular groove. Without limiting assumptions and complex mathematical concepts, the investigated method utilizes a numerical mode-matching methodology to obtain the equivalent magnetic current on the groove. A mode-by-mode substitution from the field expansion outside and inside of the groove for each pair of mode expansion coefficients leads to a linear system of equations. For this purpose, an efficient integration method is used to numerically calculate the constructed, highly oscillatory singular integral. The results are in good agreement with other numerical methods. By evaluating the above results, it can be concluded that this approach is accurate for cases of both narrow and wide grooves. The simulation time for several techniques was measured, and the proposed approach has acceptable time efficiency, especially for wide grooves.

\section{REFERENCES}

[1] W. H. Choi, H. K. Jang, J. H. Shin, T. H., Song, J. K. Kim, and C. G. Kim, "Monostatic RCS reduction by gap-fill with epoxy/MWCNT in groove pattern," Journal of the Korean Institute of Electromagnetic and Science, vol. 12, no. 1, pp. 101106, 2012.

[2] M. Bozorgi, A. Tavakoli, G. Monegato, S. H. H. Sadeghi, and R. Moini, "Backscattering from a two dimensional rectangular crack using FIE," IEEE Transactions on Antennas And Propagation, vol. 58, no. 2, pp. 552-564, 2009.

[3] K. Barkeshli and J. L. Volakis, "Scattering from narrow rectangular filled grooves," IEEE Transactions on Antennas and Propagation, vol. 39, no. 6, pp. 804-810, 1991.

[4] T. Van and A. W. Wood, "Finite element analysis of electromagnetic scattering from a cavity," IEEE Transactions on Antennas and Propagation, vol. 51, no. 1, pp. 130-137, 2003.

[5] S. S. Bindiganavale and J. L. Volakis, "A hybrid FE-FMM

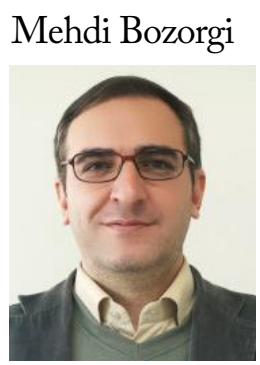

was born in Isfahan, Iran, on September 4, 1977. In 2018, he joined Arak University, Arak, Iran, where he is currently an Assistant Professor in the Department of Electrical Engineering. His current research interests include the scattering of electromagnetic waves, electromagnetic nondestructive testing, and optics. technique for electromagnetic scattering," IEEE Transactions on Antennas and Propagation, vol. 45, no. 1, pp. 180-181, 1997.

[6] G. Bao and W. Sun, "A fast algorithm for the electromagnetic scattering from a large cavity," SIAM Journal on Scientific Computing, vol. 27, no. 2, pp. 553-574, 2005.

[7] M. A. Morgan and F. K. Schwering, "Mode expansion solution for scattering by a material filled rectangular groove," Progress in Electromagnetics Research, vol. 18, pp. 1-17, 1998.

[8] D. C. Skigin, V. V. Veremey, and R. Mittra, "Superdirective radiation from finite gratings of rectangular grooves," IEEE Transactions on Antennas and Propagation, vol. 47, no. 2, pp. 376-383, 1999.

[9] T. J. Park, H. J. Eom, and K. Yoshitomi, "An analysis of transverse electric scattering from a rectangular channel in a conducting plane," Radio Science, vol. 28, no. 5, pp. 663-673, 1993.

[10] Y. H. Cho, "TM plane-wave scattering from finite rectangular grooves in a conducting plane using overlapping Tblock method," IEEE Transactions on Antennas and Propagation, vol. 54, no. 2, pp. 746-749, 2006.

[11] J. A. Crow, "Quadrature of integrands with a logarithmic singularity," Mathematics of Computation, vol. 60, pp. 297301,1993.

[12] M. Carley, "Numerical quadratures for singular and hypersingular integrals in boundary element methods," SIAM Journal on Scientific Computing, vol. 29, no. 3, pp. 12071216, 2007.

[13] S. Xiang and W. Gui, "On generalized quadrature rules for fast oscillatory integrals," Applied Mathematics and Computation, vol. 197, no. 1, pp. 60-75, 2008.

[14] G. V. Milovanovic and M. P. Stanic, "Numerical integration of highly oscillating functions," in Analytic Number Theory, Approximation Theory, and Special Functions. New York, NY: Springer, 2014, pp. 613-649.

[15] G. V. Milovanovic, "Computing integrals of highly oscillatory special functions using complex integration methods and gaussian quadratures," Dolomites Research Notes on Approximation, vol. 10(special issue), pp. 79-96, 2017. 